\title{
Reaktionen auf die westeuropäische Wirtschaftsintegration in Ostmitteleuropa: Die Tschechoslowakei und Polen von den fünfziger bis zu den siebziger Jahren
}

\author{
Dagmara JAJEŚNIAK-QUAST
}

\section{Einführende Fragestellung}

Der vorliegende Beitrag über die Reaktionen auf die westeuropäische Wirtschaftsintegration in Ostmitteleuropa am Beispiel Polens und der Tschechoslowakei fragt, wie die westeuropäischen Integrationsprozesse in Ostmitteleuropa wahrgenommen wurden und welche Effekte sich durch diesen Prozess für die Handelsbeziehungen Polens und der Tschechoslowakei zu den westeuropäischen Ländern vom Beginn der fünfziger bis zum Zeitpunkt der ersten Erweiterung der Gemeinschaft im Jahre 1973 ergaben. ${ }^{1}$

Dabei beschränkt sich diese Untersuchung allerdings nicht auf die Länder der Europäischen Wirtschaftsgemeinschaft (EWG), sondern versteht die Integrationsprozesse Westeuropas in einem breiten historischen Kontext. Unter „Integration“ wird die schrittweise Abschaffung ökonomischer Schranken zwischen unabhängigen Staaten und die zunehmende Verflechtung von deren Volkswirtschaften, sowie das Zusammenwachsen der Güter- und Faktormärkte verstanden. ${ }^{2}$ Dieser Prozess vollzog sich nicht nur in der Zollunion im Rahmen der EWG, sondern auch in der Freihandelszone im Rahmen der European Free Trade Association (EFTA) sowie im Fall der Nordeuropäischen Länder im Rahmen des NORDEK. Diese alternativen Integrationsmodelle entstanden zum Teil als Reaktion auf die Entstehung der EWG und wirkten in gegenseitiger Konkurrenz. Gerade diese Vielfalt - so eine These - versuchten die ostmitteleuropäischen Länder für die Durchsetzung eigener Interessen zu nutzen.

Schon jetzt kann man sagen, dass der Eiserne Vorhang nicht immer so undurchlässig war wie ursprünglich angenommen. Besonders ab den 1970er Jahren flossen verstärkt westliches Kapital und Technologien in Form von Krediten und Patentlizenzen nach Ostmitteleuropa. Zum Beispiel erwarb Polen drei Viertel aller westlichen Lizenzen seit dem Ende des Zweiten Weltkrieges in der Zeit von 1971-1979. Ab Mitte der achtziger Jahre kamen zu den Portfolioinvestitionen auch einzelne Joint-Ventures, also ausländische Direktinvestitionen aus den EWG-Ländern in der Region. ${ }^{3}$ Vor diesem Hintergrund gilt es die These kritisch zu hinterfragen, wonach

1. Für zahlreiche Hinweise zu diesem Text danke ich Herrn Prof. André Steiner, Frau Prof. Helga Schultz und Herrn Dr. Uwe Müller.

2. Vgl. dazu: H.-J. WAGENER, T. EGER, H. FRITZ, Europäische Integration. Recht und Ökonomie, Geschichte und Politik, Verlag Franz Vahlen, München, 2006, S.35 ff. 
die wirtschaftlichen Beziehungen zwischen den RGW [Rat für gegenseitige Wirtschaftshilfe]- und westeuropäischen Ländern seit dem Zweiten Weltkrieg vollständig abgebrochen waren. Der vorliegende Beitrag zeigt, dass es ein Spannungsfeld zwischen der politisch motivierten Ablehnung der westeuropäischen Integration in den sozialistischen Staaten und dem wirtschaftlich bedingten Bestreben einiger Staaten gab, mit den EWG- und EFTA-Mitgliedsländern Handelskontakte zu unterhalten. Trotz der Nationalisierung des Bankwesens nach dem Zweiten Weltkrieg wurden sowohl in Polen als auch in der Tschechoslowakei zwei Banken nicht vollständig verstaatlicht, die den Außenhandel mit westlichen Ländern abwickeln sollten. Bis 1989 bleibt die Bank Handlowy [Handelsbank] in Polen - neben dem polnischen Staat als größtem Gesellschafter - auch teilweise in ausländischer, vorwiegend westeuropäischer Hand. Die ausländischen Beteiligungen erleichterten den Finanzverkehr insbesondere mit Unternehmen aus Westeuropa und den USA. Die westlichen Unternehmen, die weiterhin mit Polen Kontakte pflegten, vertrauten diesen Banken mehr als den rein staatlichen Finanzinstitutionen. Eine ähnliche Situation war in der Tschechoslowakei festzustellen. Dort übernahm die Gewerbebank [Živnostenská banka] in der gesamten sozialistischen Periode eine Vermittlerrolle im Zahlungsverkehr mit dem westlichen Ausland. ${ }^{4}$ An dieser Bank gab es auch eine Minderheitsbeteiligung von ausländischem Kapital.

\section{Forschungsstand und Methoden}

Die wirtschaftspolitischen Reaktionen in den ostmitteleuropäischen Ländern auf die wirtschaftliche Integration des westlichen Teils des Kontinents und die daraus folgenden realwirtschaftlichen Konsequenzen in Ostmitteleuropa sind bis heute nicht ausreichend erforscht. Die zeitgenössische Literatur zum Thema der wirtschaftlichen Integration Westeuropas fällt in Polen und der Tschechoslowakei (ČSSR) eher spärlich aus. Der wichtigste Grund dafür ist, dass dieser Prozess in beiden Ländern in der sozialistischen Periode offiziell abgelehnt und verurteilt wurde. Jan Kułakowski - der erste Unterhändler Polens in den Assoziierungsverhandlungen mit der Europäischen Union - erinnert sich, dass in den 1950er Jahren ein Engagement für die pro-europäische Bewegung nicht möglich war und schon das Interesse an den entstehenden europäischen Strukturen Kritik hervorrief. ${ }^{5}$ Bis in die 1970er Jahre hinein und damit bis zu der neuen auf Entspannung und Verständigung ausgerichteten Ostpolitik der meisten EWG-Länder, sahen die Ent-

3. Siehe dazu: Á. TÖRÖK, Á. GYÖRFFY, Ungarn in der Vorreiterrolle, in: J. GÜNTHER, D. JAJEŚNIAK-QUAST (Hrsg.), Willkommene Investoren oder nationaler Ausverkauf? Ausländische Direktinvestitionen in Ostmitteleuropa im 20. Jahrhundert, Berliner Wissenschaftsverlag, Berlin, 2006, S.253-274.

4. JIRÁSEK, Zdeněk/MAŁKIEWICZ, Andrzej (2005): Polska i Czechosłowacja w dobie stalinizmu (1948-1956) [Polen und die Tschechoslowakei in der Zeit des Stalinismus (1948-1956)], Warszawa: Instytut Studiów Politycznych Polskiej Akademii Nauk, S. 182 und 184. 
scheidungsträger der Volksrepublik (VR) Polen und der ČSSR den Integrationsprozess des Westens als eine Konzentration des Kapitalismus an, die maßgeblich durch die USA inspiriert sei. Spätestens seit Anfang der 1950er Jahre, also mit dem Beginn des westlichen Wirtschaftsembargos gegenüber Ostmitteleuropa, wurde daher dieser Prozess offiziell abgelehnt. In beiden Ländern wurden derartige Kontakte zum westlichen Teil des Kontinents in den 1950er Jahren auch als Landesverrat angesehen. In der Tschechoslowakei wurden besonders viele Wirtschaftsexperten während der politischen Schauprozesse der 1950er Jahre zum Tode verurteilt. ${ }^{6}$

Eine erste Monographie über die Tendenzen und Perspektiven des Handels zwischen Polen und den Ländern der EWG erschien in Polen erst im Jahre 1974. ${ }^{7}$ Mit dem Außenhandel und den wirtschaftlichen Beziehungen zwischen den beiden Blöcken befasste sich die Forschung systematisch erst Ende der achtziger Jahre. ${ }^{8}$ Anders sieht es in den zahlreichen wirtschaftlichen Fachperiodika aus, die für die zu untersuchende Zeitperiode allein in Polen mit ca. 218 Titeln beziffert werden können. In Polen diskutierte man die Auswirkungen der westlichen Integration auf den polnischen Außenhandel und die Binnenwirtschaft für den hier betrachteten Zeitabschnitt in unregelmäßigen Abständen in „Ekonomista“ [,Ö̈konom“] (Warszawa 1947-2000), „Handel Zagraniczny“ [Außenhandel] (Warszawa: 1955-1984) und Życie gospodarcze“ [Wirtschaftsleben] (Katowice: 1945-1949, Warszawa 1949-1988). Für die Tschechoslowakei sind in diesem Kontext die zahlreichen Veröffentlichungen in den zeitgenössischen Periodika wie „Plánované hospodárství“ [„Planwirtschaft"] oder „Hospodářské noviny“ [,Wirtschaftsnachrichten“] sowie „Zahraniční obchod"[Außenhandel] vor allem in der Zeit des Prager Frühlings von großem Interesse. ${ }^{9}$ Bis heute sind allerdings Untersuchungen zu diesem Thema, mit Ausnahme von wenigen ersten Ansätzen, rar geblieben. ${ }^{10}$

5. KUŁAKOWSKI, Jan: Polityka Polski wobec Unii Europejskiej [Politik Polen bzgl. der Europäischen Union], in: Sprawy Międzynarodowe rocznik 1997 [Jahrgang 1997], unter: http:// www.sprawymiedzynarodowe.pl/rocznik/1997/

jan_kulakowski_polityka_polski_wobec_unii_europejskiej.html

6. Vgl. K. KAPLAN, Komunistický rezim a politické procesy v Československu [Das kommunistische Regime und die politischen Prozesse in der Tschechoslowakei], Barrister \& Principal, Brno 2001; Id., Nekrvavá revoluce [Die unblutige Revolution], Mladá fronta, Praha, 1993; Id., Die politischen Prozesse in der Tschechoslowakei 1948-1954, Oldenbourg, München, 1986.

7. Vgl.: A WIECZORKIEWICZ, Tendencje i perspektywy handlu między Polską a krajami EWG [Tendenzen und Perspektiven des Handels zwischen Polen und den Ländern der EWG], Państwowe Wydawnictwo Naukowe, Warschau, 1974.

8. Vgl.: M. HAENDCKE-HOPPE (Hrsg.), Außenwirtschaftssysteme und Außenwirtschaftsreformen sozialistischer Länder. Ein intrasystemarer Vergleich, Duncker \& Humblot, Berlin, 1988.

9. J. PLEVA, Konference o plánování zahraničního obchodu [Die Konferenz über die Planung des Außenhandels], in: Plánované hospodářství, 2(1968); Z. ŠEDIVÝ, Ke koncepci rozvoje vnăjších ekonomických vztahů [Zur Konzeption der Entwicklung der Außenwirtschaftsbeziehungen], in: Plánované hospodářství, 7(1968); K. PODLAHA, Struktura vnăjších hospodářských vztahů a ekonomická rovnováha [Die Struktur der Außenwirtschaftsbeziehungen und das wirtschaftliche Gleichgewicht], in: Plánované hospodáøství, 4(1969); J. KUBÁLEK, Konkretizace hospodářské politiky v oblasti vnijššch ekonomických vztahů [Die Konkretisierung der Wirtschaftspolitik im Bereich der Außenwirtschaftsbeziehungen], in: Hospodářské noviny, 09.08.1968. 
Die Erforschung der Wirtschaftsbeziehungen zwischen den westeuropäischenund einzelnen RGW-Ländern im Kalten Krieg bleibt also nach wie vor eine anspruchsvolle Aufgabe. Derartige Untersuchungen wurden parallel von Forschergruppen initiiert, die von Alice Teichová, Dieter Stiefel und Gertrude Enderle-Burcel in Wien, Luciano Segreto in Florenz sowie André Steiner in Potsdam geleitet werden. Darüber hinaus gibt es einzelne neuere Veröffentlichungen zu diesem Thema. ${ }^{11}$ Für die Tschechoslowakei sind vor allem Beiträge von Jaroslav Kučera, ${ }^{12}$ Christoph Buchheim, ${ }^{13}$ Bohumír Brom ${ }^{14}$ und Lenka Málková ${ }^{15}$ zu nennen.

Die Auswertung der Primärquellen in den Nationalarchiven ist auf Grund des geschilderten Forschungsstandes unumgänglich. Außerdem werden die Statistiken des Außenhandels herangezogen. Meine Recherchen basieren vor allem auf den Quellen aus den Zentralen Archiven in Warschau und Prag: Prager Staatsarchiv (Státní ústřední Archiv, SÚA), Warschauer Archiv der Neuen Akten (Archiwum Akt Nowych) sowie Archiv des Außenministeriums (Archiwum MSZ). Für das Thema sind in Prag insbesondere die Akten des Außenhandelsministeriums (Ministerstvo zahraničního obchodu) sowie die Akten der Wirtschaftskommission (Ekonomická komise) und die Bestände der Staatlichen Planbehörde (Statní úřad plánování, SÚP) von großer Bedeutung. Für die polnische Seite erfasste ich vor allem die Bestände der Plankommission (Państwowa Komisja Planowania Gospodarczego PKPG bzw. Komisja Planowania przy Radzie Ministrów), des Ministeriums für Industrie und Handel (Ministerstwo Przemysłu i Handlu, MpiH),

10. Die ersten Ansätze dieses Themas findet man auch in dieser Zeitschrift. Siehe für Polen: A. KROK-PASZKOWSKA, J. ZIELONKA, Poland's Road to the European Union, in: Journal of European Integration History, 2(2004), S.7-24, hier: 11-13 und für die Tschechoslowakei: J. KARALAS, P. KRATOCHVÍL, Czechoslovakialthe Czech Republik and European Integration: During and After the Cold War, in: idem., S.25-42, hier: 26-32; sowie: J. SCHEVARDO, Der Westen im Osten. Reaktion auf die westeuropäische Wirtschaftsintegration in Ostmitteleuropa, [unveröffentlichtes Manuskript], Zentrum für Zeithistorische Forschung, Potsdam, 2005.

11. J. ELORANTA, J. OJALA (eds), East-West Trade and the Cold War, Jyväskylä Studies in Humanities, Jyväskylä, 2005.

12. J. KUČERA, Reformdynamik und wirtschaftspolitischer Alltag. Der Außenhandel in Theorie und Praxis der tschechoslowakischen Wirtschaftsreform der sechziger Jahre, in: Ch. BOYER (Hrsg.), Sozialistische Wirtschaftsreformen. Tschechoslowakei und DDR im Vergleich, Klostermann, Frankfurt am Main, 2006, S.311-355.

13. Ch. BUCHHEIM, Die Integration der Tschechoslowakei in den RGW. In: Bohemia, in: Zeitschrift für die Geschichte und Kultur der böhmischen Länder, 1(2001), S.1-10.

14. B. BROM, Dokumenty z českých archívů k historii mezinárodných hospodářských vztahů v období studené války: studie o pramenech [Dokumente aus den Tschechischen Archiven für die Geschichte der internationalen Wirtschaftsbeziehungen zur Zeit des Kalten Krieges: Eine Studie über die Archivquellen], Karolinum, Prag, 2002; ders.: Československo a západoevropská integrace (základní rysy vzájemných hospodárských, zejména obchodních vztahů od konce 2. svătové války do počatku 90. let) [Die Tschechoslowakei und die westeuropäische Integration (Grundrisse der gegenseitigen Handelsbeziehungen seit dem Ende des 2. Weltkrieges bis Anfang der 1990er Jahre], [unveröffentlichtes Manuskript], Staatliches Zentralarchiv, Prag, 2000.

15. L. MÁLKOVÁ, Vývoj spolupráce mezi Evropskými společenstvími a Československem do roku 1992 [Die Entwicklung der Beziehungen zwischen der Europäischen Union und der Tschechoslowakei bis 1992], unveröffentlichte Magisterarbeit, Wirtschaftshochschule Prag, 1999. 
des Außenhandelsministeriums (Ministerstwo Handlu Zagranicznego) und des Zentralkomitees der Polnischen Vereinigten Arbeiterpartei (Komitet Centralny PZPR).

\section{Phasen der Wahrnehmung der westeuropäischen Integration in Ostmitteleuropa}

Die Wahrnehmung und Bewertung der westeuropäischen Wirtschaftsintegration in Polen und der Tschechoslowakei war - ähnlich wie die tatsächlichen Handelsbeziehungen - nicht statisch, sondern unterlag verschiedenen historischen Einflüssen. Ich beschränke mich zunächst auf eine Periodisierung des Hauptdiskurses, ohne dabei auf die spezifischen Unterschiede zwischen Polen und der Tschechoslowakei einzugehen. In der Wahrnehmung der Integrationsprozesse wird vor allem der Unterschied zwischen den einzelnen Jahrzehnten deutlich. Die Reaktion auf die westeuropäische Wirtschaftsintegration in Ostmitteleuropa ist trotz der offiziellen Ablehnung dieses Prozesses durch die kommunistischen Parteien sehr vielschichtig. Das Beispiel der Tschechoslowakei und Polens in der Zeit von den fünfziger bis zu den siebziger Jahren zeigt, dass diese Reaktionen von der völligen Abschottung, von Autarkiebestrebungen, über die eigene Integration im Rahmen des RGW bis hin zu gezielten Bemühungen um den Dialog mit den Integrationsbündnissen der westeuropäischen Länder reichen.

Es wäre eine Binsenwahrheit zu sagen, dass die sozialistischen Länder der Entwicklung der wirtschaftlichen Beziehungen mit den Ländern Westeuropas, darunter mit den Staaten der EWG, eine immer größere Bedeutung beimaßen. Aus der zeitgenössischen polnischen Sicht sprachen für diese Beziehungen eine große Konvergenz der Struktur der Importnachfrage der sozialistischen Länder (vor allem Investitionsgüter) mit der Struktur des Exportangebots der EWG-Länder, die große Aufnahmefähigkeit des EWG-Marktes, die historisch bedingte Tradition des gemeinsamen Handels, die geographische Nähe, woraus relativ niedrige Transportkosten resultierten, und schließlich das wachsende Interesse der EWG-Länder an der Erweiterung der wirtschaftlichen Beziehungen mit den sozialistischen Staaten. ${ }^{16}$

Die historische Tradition des gemeinsamen Handels wird durch den Anteil der westlichen Staaten und den USA im Außenhandel mit Polen und der Tschechoslowakei vor dem Krieg deutlich. Dieser Anteil verringerte sich von durchschnittlich 85\% bzw. $90 \%$ vor dem Zweiten Weltkrieg und entsprechend $60 \%$ bzw. 68\% noch im Jahre 1948 auf durchschnittlich 33\% bzw. 30\% im Jahre 1952 (siehe Abbildung $1)$.

16. Vgl. dazu: Z. KAMECKI, Integracja gospodarcza $w$ Europie Zachodniej a handel Wschód-Zachód [Die Wirtschaftsintegration in Westeuropa und der Ost-West Handel], Sprawy Międzynarodowe, Warszawa, 10-11(1970), S.30-43, hier: 30. 
(1) Anteil der westeuropäischen Staaten und der USA im Außenhandel Polens und der Tschechoslowakei vor und nach dem Zweiten Weltkrieg (in Prozent)

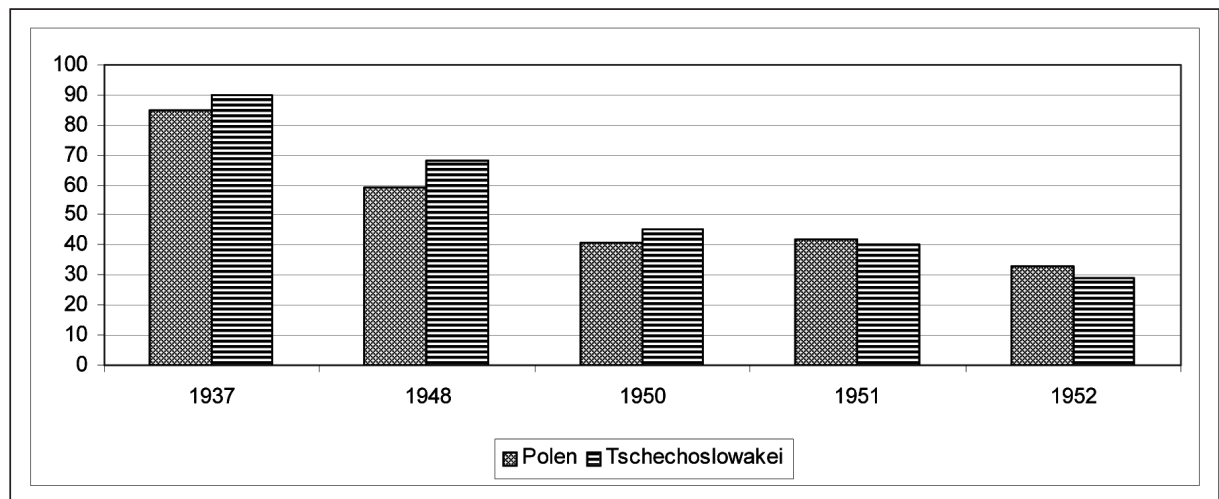

Quelle: S. BIALER, O międzynarodowych stosunkach ekonomicznych nowego typu [Über die internationalen Wirtschaftsbeziehungen des neuen Typs], in: Ekonomista, III(1953), S.56.

\subsection{Die späten 1940er und 1950er Jahre}

Direkt nach dem Krieg waren die ostmitteleuropäischen Wirtschaftswissenschafter noch unsicher, ob es sich bei der westeuropäischen Wirtschaftsintegration um einen dauerhaften Trend handelt. Die Zusammenschlüsse zur Zoll- und Wirtschaftsunion wurden als eine Mode bezeichnet. Schon bald standen die ostmitteleuropäischen Wirtschaftsexperten diesen Prozessen allerdings sehr skeptisch gegenüber. Das Hauptargument gegen die Zoll- und Wirtschaftsunion war, dass die damit verbundenen Integrationsprozesse nur den Wirtschaften weniger westeuropäischer Kernländern Vorteile bringen. ${ }^{17}$ Eine Zollunion lehnte man im Osten des Kontinents kategorisch ab, denn eine Zollunion (wie die EWG) reduziert bzw. hebt im Unterschied zur Freihandelszone (wie die EFTA) nicht nur die Zollgrenzen innerhalb der Gemeinschaft auf, sondern erhebt auch einen gemeinsamen Außenzoll. Ein gemeinsamer Außenzoll würde demnach die ostmitteleuropäischen Länder, die traditionell mit Westeuropa Handel betrieben, sehr stark benachteiligen.

Nach der Unterzeichnung der Römischen Verträge im März 1957 wurde die Zollunion von sechs westeuropäischen Ländern schrittweise zur Realität und damit erfuhren auch die ostmitteleuropäischen Vorbehalte eine Bestätigung. Im Angesicht des Kalten Krieges wurden die gesamteuropäischen Wirtschafts- und Handelsbeziehungen, sowohl im Osten als auch im Westen Europas, immer mehr durch den Austausch in jeweils regionalen wirtschaftlichen Gruppierungen (RGW vs. EWG/ EFTA) ersetzt. Diese Integrationsorganismen in Ost und West entwickelten sich vor

17. Włosko-francuska unia celna [Die italienisch-französische Zollunion], in: Życie Gospodarcze, 3(1948), S.118-119, hier: 119. 
allem in den 1950er Jahren in einer völligen gegenseitigen Isolation. Unter diesen Bedingungen führten die europäischen Integrationsprozesse - trotz der Wachstumsstimuli für die integrierten Länder - in der Perspektive der gesamteuropäischen Arbeitsteilung zu eindeutig negativen Ergebnissen.

Ursprünglich sahen die ostmitteleuropäischen Ökonomen den Handel zwischen Ost- und Westeuropa als eine Notwendigkeit für beide Seiten an. Bereits kurz nach dem Zweiten Weltkrieg schrieben polnische Wirtschaftswissenschaftler darüber, dass die Okkupationszonen in Westdeutschland, vor allem die Bi-Zone, nicht das ursprüngliche ökonomische Potenzial erreichen könne, wenn sie weiterhin von der sowjetischen Besatzungszone (SBZ) und den Ländern Osteuropas abgekoppelt blieben. ${ }^{18}$

Der „amerikanische Imperialismus“ war in den Augen der osteuropäischen Experten dafür verantwortlich, dass die westeuropäischen Länder die Möglichkeit des Bezugs von Lebensmitteln aus den alten Herkunftsländern Ostmitteleuropas einbüßten. ${ }^{19}$ Hier wurden vor allem der „Battle Act“ und andere Begrenzungen im Rahmen des Marshall-Plans genannt. So gelten die Restriktionen der Mutual Security Agency (MSA) oder die Arbeit der Consultive Group Cooperation Committee (CGCOCOM) als Beispiele in dieser Hinsicht. ${ }^{20}$ Die Rolle der USA bei der Zerstörung der traditionellen Ost-West-Wirtschaftsbeziehungen und ihr Einfluss im Integrationsprozess Westeuropas wurde aber nicht nur von den ostmitteleuropäischen Wissenschaftlern und Politikern betont, sondern auch im Westen thematisiert. So wählte der Schwede Gunnar Adler-Karlsson in seiner bekannten „Abrechnung“ mit den Ost-West-Wirtschaftsbeziehungen bewusst eine ähnliche Argumentation, als er feststellte, dass der amerikanische Kongress den Handel zwischen Ost- und Westeuropa unterbinden wollte, um damit die innere Wirtschaftskraft der Sowjetunion ernsthaft zu schädigen. ${ }^{21}$

Die Schuld für das Abbrechen der Handelskontakte zwischen Ost- und Westeuropa gaben die ostmitteleuropäischen Wirtschaftspolitiker eindeutig der Politik der USA und dem fortschreitenden Integrationsprozess Westeuropas, mit dessen einzelnen Schritten wie der Gründung der Organisation für europäische Wirtschaftszusammenarbeit (Organisation for European Economic Cooperation - OEEC, 1948), der Europäischen Zahlungsunion (1950), der Europäischen Gemeinschaft für Kohle und Stahl (1951) bis hin zu den Römischen Verträgen im Jahre 1957. ${ }^{22}$ Der Integrationsprozess wurde wiederum als entscheidend für die Reaktivierung

18. F. WILIŃSKI, Bizonia [Die Bizone], in: Życie Gospodarcze, 5(1948), S.194-197, hier: 197.

19. Siehe dazu: A. PAWLIKIEWICZ, Destrukcyjne skutki planu Marshalla [Die destruktiven Folgen des Marshallplans], in: Życie Gospodarcze, 5(1950), S.235-238, hier vor allem: 238.

20. Im Jahre 1951 beschloss der Kongress der USA das Battle Act, das mit dem Rückzug der wirtschaftlichen Hilfe der USA für die westeuropäischen Länder drohte, die weiterhin Handelsbeziehungen, vor allem den Handel mit den Embargowaren, mit sozialistischen Ländern unterhalten. Die MSA wurde im Jahre 1952 ins Leben gerufen und kontrollierte die Marshallplanländer. Ebenfalls im Jahre 1952 wurde das C.G. CO. COM mit Sitz in Paris zum Zweck der Kontrolle des Handels mit Osteuropa gegründet.

21. G. ADLER-KARLSSON, Der Fehlschlag. 20 Jahre Wirtschaftskrieg zwischen Ost und West, Europa Verlag, Wien, 1971, S.123. 
des „deutschen Imperialismus“ und die Exportexpansion der Bundesrepublik von den ostmitteleuropäischen Politikern und Wirtschaftsexperten angesehen und gefürchtet. ${ }^{23}$ Vor allem wurde der so genannte Schuman-Plan scharf verurteilt, denn in ihm sahen die ostmitteleuropäischen Experten eine wesentliche Unterstützung für westdeutsche Eisen- und Stahlunternehmen. Daher wurde der Schuman-Plan als ein Werkzeug der Aggressionspolitik der großen Stahlmonopole der USA und der westdeutschen „Magnaten aus dem Ruhrgebiet ${ }^{“ 24}$ angesehen und als eine „Party der amerikanischen Monopolisten unter dem Namen Schumanplan“25 bezeichnet. Die Aufhebung der alliierten Kontrolle über die Produktion von Kohle und Stahl, die Reaktivierung der Vorkriegskartelle und die Steigerung der Rüstungsproduktion in Westdeutschland sah man in Ostmitteleuropa als eine direkte Folge des ,atlantischen Integrationsprozesses“ ${ }^{\text {“26 }}$ Westeuropas.

Die steigende Exportkraft Westdeutschlands befand sich vor allem mit der auf Export orientierten tschechoslowakischen Wirtschaftspolitik auf Kollisionskurs. Schon unmittelbar nach dem Zweiten Weltkrieg hoffte unter anderem Präsident Edvard Beneš, dass nach der Kriegsniederlage Deutschlands die Tschechoslowakei als eines der am meisten industrialisierten Länder Ostmitteleuropas die einstige Rolle Deutschlands als Lieferant von industriellen Produkten für den europäischen Markt, vor allem von Erzeugnissen der Schwerindustrie, übernehmen könnte. ${ }^{27}$

22. K. PIOTROWSKA-HOCHFELDOWA, Europejska Wspólnota Gospodarcza [Die Europäische Wirtschaftsgemeinschaft], in: Ekonomista, 1(1958), S.165-188.

23. Vgl.: M. TOMALA, Odradzanie się ekspansji ekonomicznej monopoli zachodnio-niemieckich [Das Erneuern der wirtschaftlichen Expansion der westdeutschen Monopole], in: Ekonomista, 1(1955), S.134-142 und ders: Besprechung des Buches von K. H. Domdey: Die deutschen Monopole auf den äußeren Märkten [Verlag Die Wirtschaft, Berlin, 1959], in: Ekonomista, 4-5(1959), S.1095-1099, hier: 1098. Noch stärker wurde das Problem des ,amerikanischen Programms der Wirtschaftsintegration Westeuropas“ und der Verknüpfung der Zoll- und Wirtschaftspolitik Westdeutschlands mit der USA-Politik in der DDR-Literatur diskutiert, was die polnische Fachliteratur rezipierte: J. RUTKOWSKI, Besprechung des Sammelbandes: Zur Finanzpolitik der Deutschen Bundesrepublik. I. Anton Borgmeier: Preispolitik, II. Rolf Lohse: Die Zollpolitik [Berlin: Verlag die Wirtschaft, 1958], in: Ekonomista, 1(1959), S. 1105-1110, hier: 1109 f.

24. A. FILIPIAK, Z obrad VI Sesji Europejskiej Komisji Gospodarczej ONZ [Aus den Verhandlungen der VI. Tagung der Europäischen Wirtschaftskommission der VN], in: Życie Gospodarcze, 15(1951), S.868-870, hier: 868.

25. L. SIENNICKI, Plan Schumana - narzędziem polityki agresji [Der Schumanplan - Werkzeug der Politik der Aggression], in: Życie Gospodarcze, 8(1951), S.470-473, hier: 473; S. BIELAK, Przed realizacja planu Schumana [Vor der Realisierung des Schuman-Plans], in: Idem., 23(1951), S.1294-1296, hier: 1294, sowie: Przemyst stalowy Francji i Europejskie Zjednoczenie Węla i Stali [Die Stahlindustrie Frankreichs und die Europäische Gemeinschaft für Kohle und Stahl], in: Idem., 1(1954), S.38-39; sowie: Czy Anglia przystapi do Europejskiego Zjednoczenia Wegla i Stali [Wird England der Europäischen Gemeinschaft für Kohle und Stahl beitreten?], in: Idem., 8(1954), S.318-320, hier: 318 .

26. Co dało Francji „Europejskie Zjednoczenie Wegla i Stalii” [Was hat Frankreich der „Europäischen Vereinigung für Kohle und Stahl” gebracht], in: Życie Gospodarcze, 18(1954), S.715-717, hier: 717; Handlarze broni zapowiadaja nowy boom [Die Waffenhändler kündigen einen neuen Boom an], in: Idem., 21(1954), S.839; W imię zapewnienia pokoju w Europie trzeba uniemoźliwić wskrzeszenie militaryzmu niemieckiego [Im Namen der Sicherung des Friedens in Europa muss man die Wiederbelebung des deutschen Militarismus verhindern], in: Idem., 24(1954), S.921-922. 
Dieses Ziel verfolgten allmählich auch die anderen ostmitteleuropäischen Länder. So wurde die wirtschaftliche Zusammenarbeit der ostmitteleuropäischen Länder als Kompensation zum Wiedererstarken des wirtschaftlichen Potenzials Deutschlands interpretiert. Der spätere Rat für gegenseitige Wirtschaftshilfe sollte das Erbe des deutschen wirtschaftlichen Einflusses im Osten des Kontinents antreten. ${ }^{28}$

\subsection{Die 1960er Jahre}

Seit Beginn der 1960er Jahre schauten die ostmitteleuropäischen Länder verstärkt über den eigenen Tellerrand und suchten nach Wegen der Nutzung der Integration zu ihrem eigenen Vorteil. Angestoßen von polnischen Wirtschaftswissenschaftlern (Włodzimierz Brus, Oskar Lange), bald gefolgt von tschechoslowakischen Ökonomen (Otta Šik) setzte eine Diskussion über die positiven Effekte wirtschaftlicher Integration ein. ${ }^{29}$ So beriefen in Polen im Jahr 1968 der Außenminister und der Minister für Außenhandel gleichzeitig eine Kommission für die Untersuchung der Möglichkeiten der Nutzung der Integrationsprozesse im Westen Europas für die polnische Volkswirtschaft ein. ${ }^{30}$

Offiziell wurden vor allem seitens der Partei die wirtschaftlichen Integrationsprozesse Westeuropas in ihren Zielen und Aufgaben weiterhin als gegen das sozialistische System gerichteter Prozess wahrgenommen. ${ }^{31}$ In den wissenschaftlichen Publikationen, die seit dem Ende der 1960er Jahre verstärkt zu dem Thema der wirtschaftlichen Integration Westeuropas in Polen publiziert wurden, sah man

27. A. ZAUBERMAN, Industrial progress in Poland, Czechoslovakia, and East Germany 1937-1962, Oxford University Press, London, u.a., 1964, S.272.

28. W. KONDERSKI, Zagadnienie wspótpracy gospodarczej państw południowo-wschodniej Europy [Das Problem der wirtschaftlichen Zusammenarbeit der Länder im süd-östlichen Europa], in: $\dot{Z} y$ cie Gospodarcze, 6(1948), S.246-249, hier: 246 f.

29. Vgl. J. SCHEVARDO, op.cit., S.7; D. JAJEŚNIAK-QUAST, Die ersten Versuche der Dezentralisierung der sozialistischen Planwirtschaft in Polen. Höhepunkte der Debatten über die Wirtschaftsreformen (1956-1968), in: H.-G. HAUPT, J. REQUATE, M. KÖHLER-BAUR (Hrsg.), Aufbruch in die Zukunft. Die 1960er zwischen Planungseuphorie und kulturellem Wandel. DDR, CSSR und Bundesrepublik Deutschland im Vergleich, Velbrück Wissenschaft, Weilerswist, 2004, S.89-106.

30. Verordnung Nr.3 des Ministers für Auswärtige Angelegenheiten vom 20.03.1962 für die Berufung einer internen Arbeitsgruppe im Außenministerium für die Angelegenheiten der Europäischen Integration und: Verordnung Nr.62 des Ministers für Außenhandel vom 13.09.1962 für die Berufung einer Arbeitsgruppe für die Bearbeitung der Folgen für den polnischen Außenhandel aufgrund des Fortschrittes der Integrationsprozesse in Westeuropa, in: A. KOCHAŃSKI, Polska 1944-1991, Informator historyczny, Tom 2, Ważniejsze akty prawne decyzje i enuncjacje państwowe (1957-1970) [Polen 1944-1991, Ein historischer Leitfaden, Band 2, Die wichtigsten Rechtsakten, Entscheidungen und Staatlichen Beschlüsse], Wydawnictwo Sejmowe, Warszawa, 2000, S.301 und 322.

31. Archiwum Akt Nowych w Warschawie [Archiv der Neuen Akten in Warschau; weiter: AAN], Komitet Centralny Polskiej Zjednoczenej Partii Robotniczej [Zentralkomitee der Polnischen Vereinigten Arbeiterpartei; weiter KC PZPR], 1354/V/101, Protokół z posiedzenia Biura Politycznego z dnia 14.03.1972 [Protokoll aus der Sitzung des Politbüros vom 14.03.1972], S.133. 
neben den ökonomischen Motiven den Integrationsprozess auch als einen politisch, ideologisch und sogar militärisch bedingten Vorgang an. ${ }^{32}$

In den Expertengremien der beiden Länder herrschte allerdings in den 1960er Jahren bereits die Meinung vor, dass die Europäische Wirtschaftsgemeinschaft den „point of no return“" erreicht hat und ,zum Erfolg verurteilt" sei. ${ }^{33}$ Angesichts des Faktes, dass die EWG in den 1960er Jahren der größte Handelsblock der Welt war und mit 185 Millionen Konsumenten den ersten Platz auf der Liste der Weltexporteure von Industriewaren einnahm, gleichzeitig auch zu den größten Importeuren von landwirtschaftlichen Produkten gehörte, war eine weitere Abnabelung der beiden Länder gegenüber dieser Gemeinschaft nicht mehr möglich.

\subsection{Die 1970er Jahre}

In den 1970er Jahren zeigte die Entspannungspolitik im Rahmen der Vorbereitung für die Konferenz für Sicherheit und Zusammenarbeit in Europa (KSZE) auch die Änderung in den wirtschaftlichen Verhältnissen zwischen den beiden Ländern und vorwiegend den EWG-Ländern. ${ }^{34}$ Im Rahmen der Vorbereitung dieser Konferenz und während des Treffens der Außenminister der Staaten des Warschauer Paktes in Budapest im Juni 1970 war dies ein Thema. Polen und andere sozialistische Länder formulierten in einem Memorandum den Vorschlag im Rahmen der KSZE auch die Probleme der Erweiterung der gesamteuropäischen Wirtschafts- und Handelsbeziehungen zu berücksichtigen. ${ }^{35}$ Dabei hatten gemeinsame Infrastrukturprojekte Priorität, wie die Schaffung eines europäischen Energiesystems, der Ausbau des Straßennetzes und die Verbesserung des Eisenbahntransportes in Europa.

Auf der anderen Seite wurde der ostmitteleuropäische Markt immer interessanter für die Länder aus den westeuropäischen Integrationsbündnissen. Vor allem nach dem Ölschock der 1970er Jahre zeigte sich die Enge des EWG-Markts und eine gewisse Sättigung. In Osteuropa (inklusive der UdSSR) bestanden dagegen große Reserven sowohl an Rohstoffen als auch an den qualifizierten Arbeitskräften. Darüber hinaus stellte Ostmitteleuropa einen großen und in keiner Weise gesättigten Konsumentenmarkt mit fast 350 Millionen Einwohnern und einem relativ groBen Wachstum dar. ${ }^{36}$ Den polnischen Wirtschaftsexperten war dieser Umstand

32. Vgl. u.a.: T. GRABOWSKI, Z. NOWAK (Hrsg.), Integracja ekonomiczna Europy Zachodniej i jej aspekty polityczno-militarne [Die wirtschaftliche Integration Westeuropas und ihre politisch-militärischen Aspekte], Instytut Zachodni, Poznań, 1969; Z.M. KLEPACKI, Zachodnioeuropejskie organizacje międzynarodowe [Die internationalen Organisationen Westeuropas], Ksiąźka i Wiedza, Warszawa, 1969.

33. M. ŁYTKO, EWG - czy tylko unia celna? [EWG - nur eine Zollunion?], in: Sprawy Międzynarodowe, 12(1968), S.48-68, hier: 56 und 64.

34. Vgl. dazu: Z. LUDWICZAK, Aktualne problemy zwiazane z Europejska Konferencja Bezpieczeństwa $i$ Wspótpracy [Die aktuellen Probleme, die die Konferenz für Sicherheit und Zusammenarbeit in Europa betreffen], in: Sprawy Międzynarodowe, 9(1970), S.23-34.

35. W. GRABSKA, Ogólnoeuropejska wspótpraca gospodarcza [Die gesamteuropäische wirtschaftliche Zusammenarbeit], in: Sprawy Międzynarodowe, 9(1970), S.35-49, hier: 48. 
bewusst. Während der vielen Treffen im Zentralkomitee der Polnischen Vereinigten Arbeiterpartei am Anfang der 1970er Jahre diskutierte man daher immer öfter die Stellung Polens bezüglich der Beziehungen zwischen den RGW-Ländern und der EWG. Man stellte fest, dass die Konsolidierungsprozesse innerhalb der EWG immer weiter voranschreiten werden. Aus der Sicht der sozialistischen Länder sollte die gemeinsame Handelspolitik der Gemeinschaft gegenüber den RGW-Ländern eine große Rolle spielen. Dabei zählte man in Polen vor allem auf die Verstärkung der industriellen Kooperation und auf westliche Kredite. ${ }^{37}$

\section{Auswirkungen der Integrationsprozesse auf den Außenhandel und Reaktionen darauf}

Eine weitere These dieses Beitrags lautet, dass gerade im Bereich des Außenhandels mit Westeuropa der Einfluss des RGW nicht sehr stark war und die Länder weitgehend eigene Interessen verfolgten. Dies lag zum einen an der institutionellen Schwäche des Rates, zum anderen daran, dass der Außenhandel im System der sozialistischen Planwirtschaft an sich einen eher niedrigen Status eingeräumt bekam. ${ }^{38}$ Diese institutionelle Schwäche des RGW führte dazu, dass sowohl Polen als auch die Tschechoslowakei immer wieder versuchten, mit westeuropäischen Ländern Handelskontakte zu unterhalten und die starren Grenzen des RGW zu überwinden. Diese Wege zu den Ländern der westeuropäischen Integrationsgemeinschaften waren sehr verschieden. Allgemein erfolgte eine Reaktion beider Länder auf die westeuropäischen Wirtschaftsintegrationsprozesse in folgenden Bereichen:

- Diskussion und Einflussnahme im Rahmen der internationalen Wirtschaftsorganisation, z.B.: der Europäischen Wirtschaftskommission (ECE) oder des Allgemeinen Zoll- und Handelsabkommens (GATT);

- Neuausrichtung der Handelbeziehungen in andere westeuropäische Märkte, vor allem im Rahmen der EFTA und NORDEK;

- „Handel“ mit den EWG-Ländern über die neutralen Staaten;

36. In den Jahren 1950-1967 war das Wachstum sowohl des Volkseinkommens als auch der Industrieproduktion der RGW-Länder höher als der EWG-Länder. Grund dafür war das niedrige Ausgangsniveau. Bei einem Ausgang der Industrieproduktion von 1950 (=100) betrug die Steigerung der Produktion im Jahre 1967 für die RGW-Länder 176 und für die EWG entsprechend 136. Siehe dazu: W. GRABSKA, Ogólnoeuropejska wspótpraca ..., op.cit., S 40.

37. AAN, KC PZPR, 1354/V/101, Protokół z posiedzenia Biura Politycznego z dnia 14.03.1972 [Protokoll aus der Sitzung des Politbüros vom 14.03.1972], S. 12 und 133.

38. Vgl. W. SEIFFERT, Das staatliche Außenhandelmonopol - Entstehungsgeschichte und Ausgestaltung bis zur Reformperiode der 1960er Jahre, in: M. HAENDCKE-HOPPE (Hrsg.), Außenwirtschaftssysteme und Außenwirtschaftsreformen sozialistischer Länder. Ein intrasystemarer Vergleich, Duncker \& Humblot, Berlin, 1988, S.11-17. 
- Nutzung der Kontakte zu den kommunistischen Parteien in Westeuropa und deren Wirtschaftsbereiche;

- Eigene Integration im Rahmen des RGW.

In diesem Beitrag wird zunächst die Neuausrichtung der Handelbeziehungen in andere westeuropäische Märkte, vor allem im Rahmen der EFTA und NORDEK und der „Handel“ mit den EWG-Ländern über die neutralen Staaten vorgestellt.

\subsection{Umlenkung der Warenströme}

Eine Möglichkeit, den Handel mit Westeuropa aufrecht zu erhalten bildeten u.a. die Umlenkungsstrategien der Warenströme. So kann auch die Steigerung der Handelsströme in die EFTA-Länder aus Polen als eine direkte Auswirkung des gemeinsamen Marktes des „kleinen Europas“ gesehen werden. Im Gegensatz zur Tschechoslowakei spielte in Polen der Außenhandel mit den späteren EFTA-Ländern schon vor dem Zweiten Weltkrieg eine große Rolle und war mit 668 Millionen Złoty Handelsvolumen im Jahre 1936 sogar höher als mit den späteren EWG-Ländern, wo diese Summe entsprechend 624 Millionen Złoty betrug. Nach dem Zweiten Weltkrieg stieg das Handelsvolumen mit den EFTA-Ländern weiter, während der Außenhandel mit den späteren EWG-Ländern bis zum Zeitpunkt der Römischen Verträge im Jahre 1957 stagnierte bzw. sank. Danach nahmen das Handelsvolumen mit beiden Integrationsblöcken zu, aber erst im Jahre 1968 übertraf der Außenhandel Polens mit den EWG-Ländern den Handel mit der EFTA.

Aus der Statistik wird ersichtlich, dass die Zollunion der EWG vor allem für die Tschechoslowakei eine große Rolle spielte, denn für sie waren die späteren EWG-Länder traditionell die wichtigsten Handelspartner. Im Fall der Tschechoslowakei, das neben der DDR am meisten industrialisierte und exportorientierte Land des RGW, brach der Handel mit diesen Ländern nach dem Zweiten Weltkrieg am stärksten ein und konnte sich von diesem radikalen Bruch erst Mitte der 1960er Jahre langsam erholen.

Auch die sich verstärkenden Handelsvolumina mit den neutralen Ländern können als eine Folge des Integrationsprozesses in Westeuropa angesehen werden. Nach der Gründung der OEEC wurden die anderen Länder Westeuropas immer interessanter für den polnischen und tschechoslowakischen Außenhandel. Skandinavien spielte dabei schon aus politischen Gründen wegen der Neutralität von Finnland und Schweden eine entscheidende Rolle. Für den finnischen Außenhandel sind Polen und die Tschechoslowakei nicht zu unterschätzende Handelspartner, denn beide Länder standen im Jahre 1955 auf dem 8. und 9. Platz des finnischen Exports und entsprechend auf dem 6. und 9. Platz bezüglich des finnischen Imports (siehe Tabelle). 
Tabelle 1: Die Haupthandelspartner Finnlands im Jahre 1955 (in Milliarden Finn Mark)

\begin{tabular}{|l|l|l|l|l|}
\hline & Export & $\begin{array}{l}\text { Ranking } \\
\text { bzgl. } \\
\text { Exports }\end{array}$ & Import & $\begin{array}{l}\text { Ranking } \\
\text { bzgl. } \\
\text { Imports }\end{array}$ \\
\hline Großbritannien & 43,5 & 1. & 35,9 & 1. \\
\hline UdSSR & 31,7 & 2. & 26,0 & 2. \\
\hline BRD & 16,5 & 3. & 15,9 & 3. \\
\hline USA & 10,4 & 4. & 9,3 & 5. \\
\hline Frankreich & 8,4 & 5. & 10,5 & 4. \\
\hline Niederlande & 8,0 & 6. & 6,4 & 7. \\
\hline Belgien/Luxemburg & 6,3 & 7. & 5,3 & 8. \\
\hline Polen & 3,6 & 8. & 8,6 & 6. \\
\hline Tschechoslowakei & 1,9 & 9. & 4,8 & 9. \\
\hline
\end{tabular}

Quelle: Handel zagraniczny Finlandii [Der Außenhandel Finnlands], Życie Gospodarcze, 8(1956), S.321.

Polen und die Tschechoslowakei beobachteten bereits seit 1952 die Integrationsbestrebungen der skandinavischen Länder im Rahmen des Nordischen Rates sehr genau. Ostmitteleuropa reagierte mit Enttäuschung auf den Beschluss der Kopenhagener Konferenz im April 1968, im Rahmen der EFTA eine nordische Gemeinschaft mit dem Ziel einer Zollunion zu gründen. Ein Jahr später - im Juli 1969 - wurde das Projekt der Nordischen Zollunion - NORDEK von den Regierungen von Dänemark, Norwegen, Schweden und Finnland umgesetzt. ${ }^{39}$ Damit entstand eine neue europäische Wirtschaftsgemeinschaft, die zwar mit ca. 22 Millionen Konsumenten und 40 Milliarden US-Dollar der gesamten Summe der BIPs viel kleiner als die EWG war (hier waren die Daten zu dem Zeitpunkt entsprechend 180 Millionen Konsumenten und 300 Milliarden US-Dollar), aber für Polen und die Tschechoslowakei bedeutete dies neue Handelsbarrieren in Europa. Immerhin betrugen die Handelsvolumina der NORDEK-Länder ca. ein Drittel des Umsatzes mit allen EFTA Ländern und entsprachen einem Fünftel des Außenhandels mit der EWG. ${ }^{40}$ Die sozialistischen Länder befürchteten darüber hinaus mit Recht, dass das langfristige Ziel der nordischen Länder darin bestehen könnte, ihre Position in

39. A. GROCHULSKI, NORDEK - nowy etap integracji krajów skandynawskich [NORDEK - die neue Etappe der Integration der skandinavischen Länder], in: Sprawy Międzynarodowe, 3(1970), S.106-116, hier: 107 und 109.

40. Ibid., S.111. 
den Verhandlungen mit der EWG zu stärken, um schließlich eine Integration in die EWG zu erreichen. Ein Hinweis für dieses mögliche Ziel waren unter anderen die Pläne für eine Festlegung der Zollsätze aller vier skandinavischen Länder auf die Höhe der EWG-Zölle.

Die Mitgliedschaft von Schweden und Finnland in der NORDEK-Gemeinschaft rief in beiden sozialistischen Länder weiterhin Sorgen hervor, dass die Neutralität der beiden Länder aufgegeben würde und sie unter die Vorherrschaft der NATO geraten könnten. Wirtschaftlich fürchteten Polen und die Tschechoslowakei die Entstehung eines weiteren abgeschotteten Wirtschaftsblocks im Norden Europas. Damit würde die bis jetzt relativ liberale Handelspolitik der nordischen Länder gegenüber den ostmitteleuropäischen Staaten aufgegeben. Für Polen hätte das den Verlust eines relativ großen wirtschaftlichen Partners bedeutet, denn der Anteil der skandinavischen Länder am polnischen Außenhandel betrug ca. 15\%. Daher schaute man mit großer Hoffnung in Richtung Finnland, wo es große Bedenken gegenüber dem NORDEK-Verbund gab. ${ }^{41}$ Gegen die Aufgabe der Neutralität, vor allem Finnlands, protestierte auch die Sowjetunion stark.

In den beiden Ländern versuchte man aber weiterhin eigene Interessen unabhängig von der Sowjetunion zu verfolgen. Bereits Mitte der 1960er Jahre herrschte unter den Wirtschaftspolitikern Polens die Meinung vor, dass die Weiterentwicklung der wirtschaftlichen Beziehungen mit den kapitalistischen Ländern eine wichtige Aufgabe sei. Sogar Piotr Jaroszewicz, der ständige Vertreter Polens im RGW, stellte fest, dass trotz der guten wirtschaftlichen Entwicklungsperspektiven Polens innerhalb des RGW, ein Teil der wirtschaftlichen Aufgaben besser erfüllt werden könnten, wenn man die Kooperation mit anderen westeuropäischen Ländern ausbauen würde. ${ }^{42}$

Die statistischen Daten bestätigen, dass sich die Handelsbeziehungen zwischen den sozialistischen und westeuropäischen Ländern seit dem Anfang der 1960er Jahre langsam normalisierten. Die Bedeutung der neutralen Staaten ist schon in der 1950er Jahren rückläufig und die der EWG-Länder steigt. Vor allem war der Zollabbau der EWG im Prozess der Normalisierung wichtig. Während der Handel Polens mit anderen Integrationsgruppen stagnierte oder sogar sank, stieg der Anteil der EWG-Länder am Außenhandel, im Export mit Polen bereits Mitte der 1950er Jahre von ca. 6\% im Jahre 1955 auf über 12\% im Jahre 1970. Der Anteil der EWG-Länder am polnischen Import stagnierte dagegen fast über den gesamten Untersuchungszeitraum bei einem Umfang von ca. 10\% (siehe Abbildung 2).

Im Fall der Tschechoslowakei setzte das Wachstum sogar früher ein. Im Gegensatz zu Polen nahm der Anteil der EWG-Länder sowohl im Fall des Exports als auch des Imports bereits seit 1953 kontinuierlich zu, von ca. 5\% im Jahre 1953 auf über 10\% im Jahre 1970. (siehe Abbildung 3).

41. Ibid., S.114.

42. P. JAROSZEWICZ, Polska, RWPG i gospodarka światowa [Polen, RGW und Weltwirtschaft], in: Sprawy Międzynarodowe, 7-8(1967), S.16-29, hier: $19 \mathrm{f}$. 
(2) Anteil des polnischen Im- und Exports aus und in die westeuropäischen Länder nach Integrationsgruppen (in Prozent)

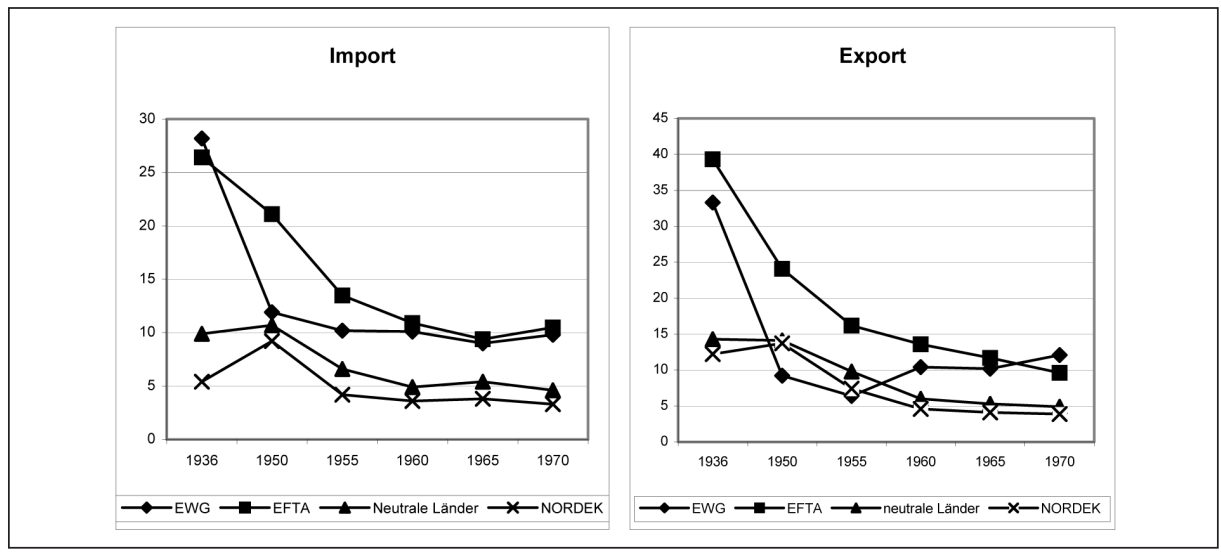

Quelle: Mały Rocznik Statystyczny 1937, S.153; Rocznik Statystyczny Handlu 1970, S.24 f.; Rocznik Statystyczny 1970, S.359 f.; Rocznik Statystyczny 1980, S.313 f.

(3) Anteil des tschechoslowakischen Im- und Exports aus und in die westeuropäischen Länder nach Integrationsgruppen (in Prozent)

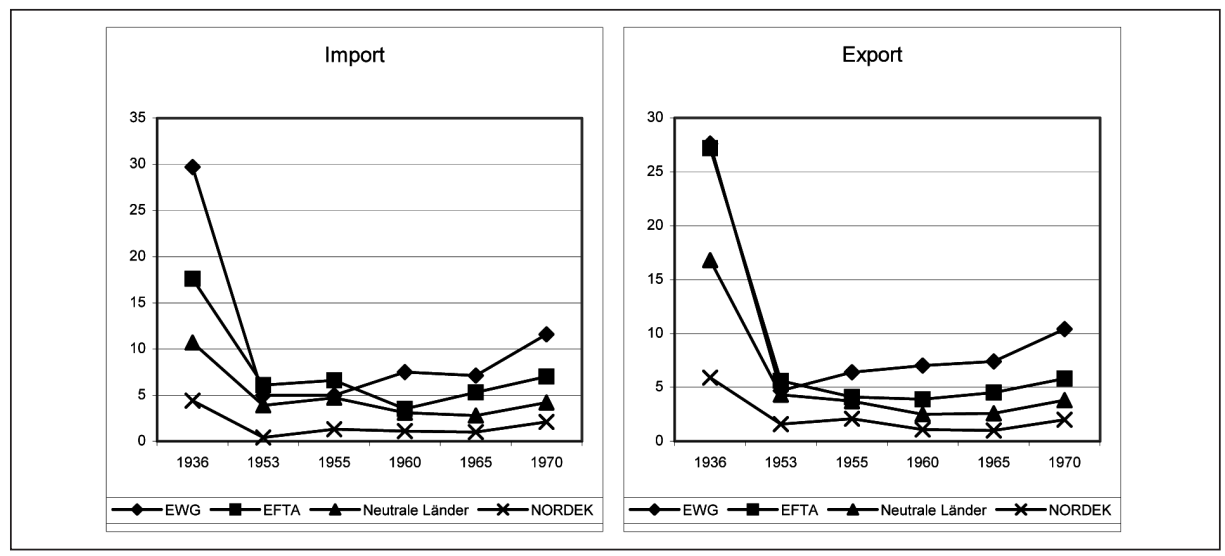

Quelle: Statistická Ročenka Republiky Československé 1938, S. 138-139; Statistická Ročenka Československé Socialistické Republiky 1960, S.361-362 und 1965, S.385 und 1968, S.421 so wie 1971, S.423 und 425-427.

\section{Zusammenfassung}

Die Wahrnehmung der westeuropäischen Wirtschaftsintegration in Ostmitteleuropa war trotz der offiziellen Ablehnung dieses Prozesses durch die kommunistischen 
Parteien nicht einseitig. Er unterlag im übrigen auch Wandlungen. Die Reaktionen auf die Integrationsprozesse im Westen des Kontinents reichten von der völligen Abschottung, über Autarkietendenzen, verstärkte Integration im Rahmen des RGW bis hin zum Dialog mit den Integrationsbündnissen der westeuropäischen Länder. Die statistischen Daten bestätigen, dass seit dem Anfang der 1960er Jahre langsam eine Ausweitung der Handelsbeziehungen zwischen den sozialistischen und westeuropäischen Ländern zu registrieren ist. Der Zollabbau der EWG spielte in diesem Prozess eine große Rolle. In den 1970er Jahren zeigte dagegen die Entspannungspolitik im Rahmen der Vorbereitung für die Konferenz für Sicherheit und Zusammenarbeit in Europa (KSZE) auch eine Änderung des wirtschaftlichen Verhältnisses der Tschechoslowakei und Polen insbesondere zur EWG.

Grundsätzlich ist festzustellen, dass die ostmitteleuropäischen Wirtschaftsexperten den Handel zwischen Ost- und Westeuropa als eine Notwendigkeit für beide Seiten ansahen. Dabei wurde die Rolle der USA in der zeitgenössischen Diskussion in ihrem Einfluss im Integrationsprozess im Westen des Kontinents und in der Zerstörung der traditionellen Ost-West-Wirtschaftsbeziehungen betont. So sprach man im Osten über einen ,atlantischen Integrationsprozess“ Westeuropas. Hier stimmen die Meinungen der ostmitteleuropäischen Wissenschaftler und Politiker mit derjenigen einiger westeuropäischer Kollegen überein.

Darüber hinaus wurde der Integrationsprozess des Westens von den ostmitteleuropäischen Politikern und Wirtschaftsexperten gefürchtet, weil er eine Reaktivierung des „deutschen Imperialismus“ und die Expansion westdeutschen Ausfuhren mit sich bringen könnte. Diese Sorge gründete natürlich in den historischen Erfahrungen Ostmitteleuropas mit dem deutschen Expansionismus.

Trotz der ideologischen Vorbehalte gegenüber den Wirtschaftskontakten mit westeuropäischen Ländern suchte die Wirtschaftspolitik Polens und der Tschechoslowakei nach Wegen, um den Kontakt zu den traditionellen Handelspartnern, den EFTA- und EWG-Ländern, aufrecht zu erhalten und damit auch einen Einfluss auf die westliche Integration auszuüben. Hier spielten die Diskussion und Einflussnahme im Rahmen der internationalen Wirtschaftsorganisation, wie der Europäischen Wirtschaftskommission (ECE) oder des Allgemeinen Zoll- und Handelsabkommens (GATT) eine große Rolle, denn das war die letzte Plattform, wo Mitglieder der beiden unterschiedlichen Integrationslager sich noch treffen konnten. Hier wurden insbesondere die negativen Folgen der Zollunion, die gemeinsame Agrarpolitik oder die Einfuhrkontingente für Ostmitteleuropa mit den westeuropäischen Ländern diskutiert.

Zeitlich variieren die Bemühungen sehr, aber bereits in den 1960er Jahren herrschte die Meinung in den Expertengremien beider Länder, dass die Europäische Wirtschaftsgemeinschaft den ,point of no return“ erreicht hat und „zum Erfolg verurteilt" sei. Angesicht der steigenden Bedeutung der EWG auf dem Weltmarkt, war eine weitere Abkopplung der beiden Länder gegenüber dieser Gemeinschaft nicht mehr möglich. Zusätzlich führte die systembedingte Schwäche des RGW auch dazu, dass sowohl Polen als auch die Tschechoslowakei immer wieder versuchten, mit den westeuropäischen Ländern Handelskontakte zu unterhalten und nach Auswegen aus den starren RGW-Grenzen zu suchen. 